\title{
Genotipificación de los polimorfismos -511, -31 y +3954 del gen de la interleucina-1 $\beta$ humana en una población colombiana con cuadro de dispepsia
}

\author{
María Teresa Arangoํ, Carlos Jaramillo', María Camila Montealegre", \\ Mabel Helena Bohórquez², María del Pilar Delgado ${ }^{1}$

\footnotetext{
${ }^{1}$ Laboratorio de Diagnóstico Molecular y Bioinformática, Departamento de Ciencias Biológicas, Facultad de Ciencias, Universidad de los Andes, Bogotá, D.C., Colombia

${ }^{2}$ Grupo de Citogenética, Filogenia y Evolución de Poblaciones, Universidad del Tolima, Santa Helena, Ibagué, Colombia
}

Introducción. Varios estudios sugieren que algunos polimorfismos del gen de la interleucina-1 $\beta$ humana (IL-1 $\beta$ ), como -511 , -31 y +3954, están asociados al cáncer gástrico, debido al efecto inhibidor que esta citocina tiene sobre la secreción ácida del estómago, lo cual facilita la colonización e infección por agentes como Helicobacter pylori, así como la génesis de estados preneoplásicos que pueden conducir al desarrollo de cáncer.

Objetivo. Genotipificar los polimorfismos $+3954,-511$ y -31 de la IL-1 $\beta$ y establecer sus frecuencias en una población de pacientes con diferente sintomatología gástrica.

Materiales y métodos. Se analizaron 111 biopsias del antro gástrico obtenidas de pacientes con sintomatología de alguna alteración gástrica. La detección de $H$. pylori en las muestras se realizó mediante PCR empleando iniciadores específicos para cada región y la genotipificación de las regiones polimórficas de la IL-1 $\beta$ se realizó por RFLP empleando las enzimas Aval, Alul y Taql para $-511,-31$ y +3954, respectivamente.

Resultados. Se detectó $H$. pylori en $59,5 \%$ de las biopsias gástricas, mientras que el estudio histopatológico reveló que $82,9 \%$ de los pacientes padecía alguna enfermedad. La caracterización de las regiones polimórficas del gen de la IL-1 $\beta$, seguida de la tipificación por RFLP, permitió evidenciar los tres posibles genotipos de cada uno de los polimorfismos en la población. En los pacientes infectados por $H$. pylori se encontró con mayor frecuencia (28,6\%) el genotipo CC en la región polimórfica -31.

Conclusión. No se encontraron diferencias significativas en los genotipos de los individuos infectados y los no infectados por $H$. pylori, a excepción del genotipo CC en la región polimórfica -31, el cual se encontró con mayor frecuencia en los pacientes con enfermedades benignas.

Palabras clave: polimorfismo de nucleótido simple, interleucina-1 beta, reacción en cadena de la polimerasa, polimorfismo de longitud del fragmento de restricción, enfermedad por Helicobacter pylori.

\section{Genetic characterization of the interleukin $1 \beta$ polymorphisms $-511,-31$ y +3954 in a Colombian population with dyspepsia}

Introduction. The human interleukin- $1 \beta$ gen (IL-1 $\beta$ ) polymorphisms such as $-511,-31$ and +3954 have been associated with the presence of gastric cancer, due to the inhibitor effect that this protein has on acid secretion in the stomach. Thisfacility can enhance the colonization and infection by agents like Helicobactor. pylori and the genesis of preneoplastic states that can lead to cancer development.

Objective. Three polymorphisms of IL-1 $\beta$ (+3954, -511 and -31$)$ will be genetically characterized and their frequencies established in a population of patients with gastric symptoms.

Materials and methods. Gastric antrum biopsies were obtained from 111 patients that showed signs of gastric disorder. A PCR was done to detect the $H$. pylori presence; a PCR using designed primers for specific regions was done to define the three polymorphic regions of IL-1 $\beta$, and a RFLP was carried out using Aval, Alul and Taql for the position $-511,-231$ and +3954 for each case.

Results. Helicobacter pylori was detected in $59.5 \%$ of the evaluated gastric while the histopathology study revealed that $82.9 \%$ of patients had some pathology. Characterization of polymorphic regions of IL-1 $\beta$ gen were joined to RFLP typing evidenced that all descfribed genotypes were present in the study population. However, patients with benign pathologies infected with $\mathrm{H}$. pylori had a high frequency of the CC genotype (28.6\%) in the -31 polymorphic regions.

Conclusion. No significant differences were found between the genotype frequenciess of the $\mathrm{H}$. pyloriinfected and the non-infected populations with one exception. The CC genotype in the -31 polymorphic region was associated with benign pathologies. 
Key words: polymorphism, single nucleotide; interleukin-1beta, polymerase chain reaction; polymorphism, restriction fragment length; Helicobacter pylori, pathology.

Helicobacter pylori es un bacilo Gram negativo microaerófilo que tiene la capacidad de establecerse en el estómago, colonizando preferencialmente las células secretoras de la mucosa estomacal (1). Se ha estimado que la infección por esta bacteria afecta a más de la mitad de la población mundial, con mayor prevalencia en los países en desarrollo (2).

Al colonizar la mucosa, $H$. pylori induce una respuesta inmunitaria, tanto celular como humoral, que conlleva a la inflamación crónica de la mucosa gástrica en la zona afectada, lo cual se ha visto asociado al desarrollo de diferentes enfermedades como gastritis, úlcera duodenal y gástrica, adenocarcinoma y linfoma gástrico $(3,4)$. En razón a que el desarrollo de la patogénesis es multifactorial, las características específicas de la cepa infecciosa, las condiciones ambientales y la genética del huésped inciden de una forma directa sobre el curso de la infección.

En el caso del agente patógeno, la presencia de factores de virulencia, como la isla patógena cag (cagPAl), la citotoxina de vacuolas (VacA) y la adhesina (BabA), entre otros factores, se ha asociado con el desarrollo de enfermedades gastroduodenales; sin embargo, existe un porcentaje de pacientes infectados con cepas de H. pylori que expresan algunos de estos factores pero que, finalmente, no desarrollan ninguna enfermedad (4). En el huésped, H. pylori induce inicialmente una respuesta inmunitaria celular, principalmente mediada por linfocitos Tayudadores, lo que estimula la producción de algunas citocinas como el factor de necrosis tumoral (FNTa), la interleucina- $1 \beta$ humana (IL-1 $\beta$ ) y otras moléculas relacionadas con la respuesta inmunitaria, como el receptor antagonista de IL-1 (IL-1RN) (4). Varios autores han descrito que algunos polimorfismos de los genes que codifican para estas citocinas, especialmente IL-1 $\beta$ y FNTa, y los niveles bajos de IL-10, parecen estar asociados al desarrollo de enfermedades gastroduodenales, cuando hay infección por $H$. pylori (3).

\footnotetext{
Correspondencia:

María del Pilar Delgado, Laboratorio de Diagnóstico Molecular y Bioinformática, Universidad de los Andes, carrera 1 № 18A-10, Bogotá, D.C., Colombia

Teléfono (571) 339 4949, extensiones 3761 a 3763

mdelgado@uniandes.edu.co

Recibido: 28/05/09; aceptado:19/11/09
}

Algunos estudios reportan una asociación entre el desarrollo de enfermedades gástricas, como el cáncer y la gastritis atrófica, y algunos polimorfismos en el gen de la IL-1 $\beta$. Entre las mutaciones descritas, se encuentran cambios puntuales 0 SNP (single-nucleotide polymorphism), ubicados en las posiciones $-511(\mathrm{C} / \mathrm{T}),-31(\mathrm{~T} / \mathrm{C}) \mathrm{y}+3954(\mathrm{C} / \mathrm{T})$ $(5,6)$. Éstos se han relacionado con aumento en la secreción de la citocina, la cual tiene un efecto estimulante sobre los linfocitos y los factores de crecimiento de las células de la mucosa gástrica $(7,8)$.

En la mayoría de los casos, la infección por H. pylori es asintomática y en algunos pacientes puede persistir durante toda la vida si no es tratada por medio de antibióticos (3). Sin embargo, un grupo de pacientes puede llegar a desarrollar enfermedades gastroduodenales benignas o malignas. Por lo tanto, es importante determinar los factores que pueden incidir en esta diferencia.

En este orden de ideas, el objetivo de este estudio fue identificar las variantes genotípicas de los polimorfismos +3954, -511 y -31 del gen de la IL$1 \beta$ humana y las frecuencias alélicas de éstos en una población de pacientes con sintomatología gástrica.

\section{Materiales y métodos \\ Población de estudio y especímenes}

Se analizaron biopsias gástricas provenientes de 111 pacientes voluntarios residentes en Ibagué, Tolima, que asistieron a la unidad de endoscopia de la Clínica Manuel Elkin Patarroyo del Instituto de Seguros Sociales de Ibagué, Colombia, los cuales accedieron a hacer parte del estudio por medio de un consentimiento informado.

Los pacientes se remitieron a la Unidad de Gastroenterología para la realización de un examen de endoscopia de vías digestivas altas, como parte del estudio por cuadro de dispepsia, durante el periodo comprendido entre marzo de 2006 y mayo de 2007.

Se excluyeron del estudio quienes presentaran enfermedades cardiovasculares 0 respiratorias, que hubiesen sido sometidos a algún tratamiento de radioterapia o quimioterapia seis meses antes de la toma de la muestra o que hubiesen ingerido algún antiácido 12 horas antes, bloqueadores de 
la bomba de protones o bloqueadores de $\mathrm{H}_{2} 15$ días antes, o que hubiesen recibido algún tipo de tratamiento antibiótico los tres meses previos a la endoscopia.

Durante el procedimiento endoscópico, el médico gastroenterólogo tomó una biopsia de la mucosa antral a todos los pacientes. Se tomaron muestras del cuerpo gástrico en aquéllos con sospecha de gastritis atrófica multifocal. De esta manera, a los pacientes que tenían nicho ulceroso se les tomaron seis biopsias, y a los demás, sólo cuatro. En quienes presentaban sólo gastritis, la biopsia se tomó para clarificar su estado, comprobar la presencia de $H$. pylori y realizar la tipificación de los polimorfismos de un solo nucleótido, o SNP, del gen de la IL-1 $\beta$ por medio de los análisis moleculares.

\section{Análisis histológico}

Los médicos patólogos de la Universidad del Tolima procesaron los cortes histológicos coloreados con hematoxilina-eosina y Giemsa, y procedieron a realizar el examen histopatológico. Los resultados fueron informados de acuerdo con los criterios del sistema Sydney modificado, según la ubicación, morfología y etiología de la lesión, y, finalmente, las lesiones se clasificaron dentro de los grupos de gastritis crónica no atrófica, gastritis atrófica, úlcera, metaplasia/displasia o cáncer, según correspondiera.

\section{Extracción de ADN}

EI ADN total fue extraído directamente de las biopsias mediante el uso del estuche Aquapure Genomic DNA Isolation de Bio-Rad®, siguiendo las recomendaciones del fabricante. La extracción se hizo en tres etapas. Se realizó la lisis del tejido usando solución tampón de lisis, la cual contiene un detergente aniónico que además inactiva las ADNasas; posteriormente, se precipitaron las proteínas por medio de sales y, finalmente, se precipitó el ADN empleando alcoholes. El ADN fue resuspendido en una solución estabilizadora para su posterior análisis molecular.

\section{Detección molecular de H. pylori}

Para detectar la presencia de $H$. pylori en las biopsias gástricas, se empleó un protocolo de PCR para la amplificación de un fragmento de alrededor de $537 \mathrm{pb}$ del gen de 16S ADNr (9). Como control positivo, se empleó la cepa de referencia NCTC11637 provista por el Instituto Nacional de Cancerología y, como blanco de reacción, agua ultrapura.
Genotipificación de los polimorfismos -511, -31 $y+3954$ del gen de IL-1 $\beta$ por medio de PCRRFLP

Posición -511. Un fragmento de 305 pb, aproximadamente, se amplificó por PCR, empleando los iniciadores 5' TGGCATTGATCTGGTTCATC 3' y 5' GTTTAGGAATCTTCCCACTT 3' (10), el cual contiene la posición polimórfica -511 que hace parte de la secuencia reconocida por la enzima de restricción Aval.

La reacción se llevó a cabo en un volumen final de $25 \mu \mathrm{l}$, que contenía $50 \mathrm{mM}$ de $\mathrm{KCl}, 20 \mathrm{mM}$ de Tris $\mathrm{HCl}$; $\mathrm{pH} 8,4,2,5 \mathrm{mM}$ de $\mathrm{MgCl}_{2}, 0,2 \mathrm{mM}$ de cada desoxinucleótido trifosfato (dNTPs), $1 \mathrm{pmol} / \mu \mathrm{l}$ de cada iniciador, 0,1 $\mathrm{U}$ de Taq polimerasa (Invitrogen TM) y $60 \mathrm{ng} / \mathrm{\mu L}$ de ADN.

El perfil térmico consistió en una desnaturalización inicial a $94^{\circ} \mathrm{C}$ por 5 minutos, seguida de 30 ciclos de un minuto a $94^{\circ} \mathrm{C}$, un minuto a $57,5^{\circ} \mathrm{C}$ y un minuto a $72^{\circ} \mathrm{C}$, y finalmente, un ciclo de extensión a $72^{\circ} \mathrm{C}$ por siete minutos. La secuencia amplificada fue sometida a un protocolo de RFLP. La reacción de restricción se llevó a cabo durante 24 horas a $37^{\circ} \mathrm{C}$, en un volumen final de $10 \mu \mathrm{l}$, que contenía:

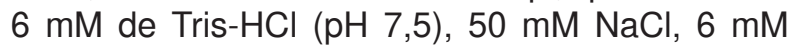
$\mathrm{MgCl}_{2}, 1 \mathrm{mM}$ de DTT, 0,1 $\mu \mathrm{g} / \mu \mathrm{l} \mathrm{BSA}, 0,2 \cup$ Aval y 5 $\mu \mathrm{l}$ del ADN amplificado.

Posición -31. Para la amplificación de la posición polimórfica -31 del gen, fue diseñado el iniciador 5' GACTCCCTTAGCACCTAGTTG 3' y, además, se empleóeliniciador5'AGAAGCTTCCACCAATACTC 3' (6). A partir de la amplificación, se obtuvo un producto de cerca de $248 \mathrm{pb}$, el cual contiene la región polimórfica -31 reconocida por la enzima de restricción Alul. La reacción se llevó a cabo en un volumen final de $25 \mu \mathrm{l}$, que contenía $50 \mathrm{mM}$ de $\mathrm{KCl}$; $20 \mathrm{mM}$ de Tris $\mathrm{HCl}$; $\left(\mathrm{pH}(8,4) ; 3,0 \mathrm{mM}\right.$ de $\mathrm{MgCl}_{2} ; 0,2$ $\mathrm{mM}$ de cada desoxinucleótido trifosfato (dNTPs); 1 $\mathrm{pmol} / \mu \mathrm{l}$, de cada iniciador; $1,25 \mathrm{U}$ de Taq polimerasa (Invitrogen TM); y $60 \mathrm{ng} / \mu \mathrm{l}$ de ADN.

El perfil térmico consistió en una desnaturalización inicial a $94^{\circ} \mathrm{C}$ por 10 minutos, seguida de cinco ciclos de $94^{\circ} \mathrm{C}$ por 30 segundos, $65^{\circ} \mathrm{C}$ por 30 segundos y $72^{\circ} \mathrm{C}$ por 30 segundos, continuando con 30 ciclos de 30 segundos a $94^{\circ} \mathrm{C}, 30$ segundos a $60^{\circ} \mathrm{C}$ y 30 segundos a $72^{\circ} \mathrm{C}$, y cinco ciclos a $94^{\circ} \mathrm{C}$ por 30 segundos, $55^{\circ} \mathrm{C}$ por 30 segundos y $72^{\circ} \mathrm{C}$ por 30 segundos, con un paso de extensión final a $72^{\circ} \mathrm{C}$ por siete minutos. A partir de la secuencia amplificada, se aplicó el protocolo de RFLP, cuya reacción se llevó a cabo durante tres horas a $37^{\circ} \mathrm{C}$, 
en un volumen final de $10 \mu \mathrm{l}$ que contenía $10 \mathrm{mM}$ de Tris- $\mathrm{HCl} ; \mathrm{pH} 7,5,10 \mathrm{mM}$ de $\mathrm{MgCl}_{2}$ y $1 \mathrm{mM}$ de ditiotreitol, una unidad de enzima y $5 \mu \mathrm{l}$ del producto amplificado (9).

Posición +3954. Se realizó la amplificación de un fragmento de $152 \mathrm{pb}$ empleando los iniciadores 5' AATTTTGCCACCTCGCCTCA 3' y 5' CGGAGCGTGCAGTTCAGTGAT 3' (6), el cual contiene la región polimórfica +3954 reconocida por la enzima de restricción Taql. La reacción se realizó en un volumen final de $25 \mu \mathrm{l}$ que contenía $50 \mathrm{mM}$ de $\mathrm{KCl}, 20 \mathrm{mM}$ de Tris $\mathrm{HCl} ; \mathrm{pH} 8,4 ; 2,5$ $\mathrm{mM}$ de $\mathrm{MgCl}_{2} ; 0,2 \mathrm{mM}$ de cada desoxinucleótido trifosfato; $1 \mathrm{pmol} / \mu \mathrm{l}$ de cada iniciador; 0,05 U de Taq polimerasa (Invitrogen TM) y $60 \mathrm{ng} / \mu \mathrm{L}$ de ADN.

El perfil térmico consistió en una desnaturalización inicial a $94^{\circ} \mathrm{C}$ por 10 minutos, seguida de 45 ciclos de 30 segundos a $94^{\circ} \mathrm{C}, 30$ segundos a $58,7^{\circ} \mathrm{C}$ y 30 segundos a $72^{\circ} \mathrm{C}$, y, finalmente, una extensión final a $72^{\circ} \mathrm{C}$ por 10 minutos. La reacción de RFLP de la secuencia amplificada se llevó a cabo durante 24 horas a $37^{\circ} \mathrm{C}$, en un volumen final de $10 \mu$ que contenía $6 \mathrm{mM}$ de Tris-HCl; pH 7,5; $50 \mathrm{mM} \mathrm{NaCl} ; 6$ $\mathrm{mM} \mathrm{MgCl} ; 1 \mathrm{mM}$ de DTT; 0,1 $\mu \mathrm{g} / \mu \mathrm{l} \mathrm{BSA} ; 0,2 \cup$ Aval y $5 \mu \mathrm{ldel}$ ADN amplificado.

\section{Visualización de los productos amplificados}

Los productos amplificados del gen 16S ADNr y del gen de la IL-1 $\beta$ fueron separados en geles de agarosa al $1,5 \%$ y $2 \%$, respectivamente; además, los productos de las digestiones enzimáticas se separaron en geles de poliacrilamida al $12 \%$. Finalmente, la visualización se llevó a cabo en el Laboratorio de Diagnóstico Molecular y Bioinformática de la Universidad de los Andes, empleando bromuro de etidio bajo luz ultravioleta.

\section{Análisis estadísticos}

Para el análisis de los datos, se empleó el programa Statistix 8, por medio del cual se realizaron pruebas de independencia de ji al cuadrado, para determinar la posible relación entre los diferentes genotipos del huésped y la presencia de $H$. pylori, tomando un valor estadísticamente significativo $p \leq 0,05$.

Además, se llevaron a cabo pruebas de comparación de frecuencias para determinar las diferencias entre las poblaciones evaluadas, en las cuales se consideró $p<0,05$ como un valor estadísticamente significativo $y$, además, se realizó la prueba de bondad y ajuste de ji al cuadrado en el programa SPSS, versión 17.0, teniendo como referencia el mismo valor de $p$ para determinar si la población en su totalidad se encontraba bajo el equilibrio de Hardy-Weinberg para cada una de las posiciones polimórficas.

\section{Consideraciones éticas}

El estudio fue aprobado por los comités de ética de las instituciones participantes. Todos los pacientes incluidos en el estudio aceptaron y firmaron el consentimiento informado.

\section{Resultados}

Se analizaron 111 biopsias de antro gástrico, provenientes de igual número de pacientes con un rango de edad de 18 a 79 años y una media de 51,49 años. De éstos, $64,6 \%$ fueron pacientes de sexo femenino y $35.4 \%$ de sexo masculino.

Como se observa en el cuadro 1, el análisis histopatológico mostró que la mayoría de las muestras evaluadas correspondieron a gastritis crónica no atrófica y úlcera gástrica acompañada de gastritis crónica superficial no atrófica $(82,9 \%)$, las cuales son enfermedades benignas asociadas a niveles de secreción normal o elevada de ácido en el estómago, mientras que los porcentajes de otras enfermedades dentro de la población evaluada fueron muy bajos (14,4\%). El 3,6\% de los pacientes presentó gastritis atrófica, el $9 \%$ metaplasia 0 displasia y el 1,8\% cáncer. Por otro lado, en 2,7\% de las muestras evaluadas no se reportó ninguna enfermedad que afectara la mucosa gástrica según el análisis histológico; por lo tanto, no fueron consideradas para los análisis posteriores. Por medio de la amplificación del gen $16 S A D N r$, se detectó la presencia de $H$. pylori en $59,5 \%$ de los pacientes con sintomatología gástrica.

La caracterización de las regiones polimórficas del gen de la IL-1 $\beta$ por medio de PCR, reveló la presencia de productos de amplificación del tamaño esperado (figura 1) y la tipificación por RFLP detectó la presencia de los tres posibles genotipos de cada uno de los polimorfismos en la población (figura 2).

Cuadro 1. Hallazgos histopatológicos.

\begin{tabular}{llrr}
\hline $\begin{array}{l}\text { Tipo de enfermedad } \\
(\%)\end{array}$ & $\begin{array}{c}\text { Diagnóstico } \\
\text { histopatológico }\end{array}$ & $\mathbf{n}$ & (\%) \\
\hline Normal & No se reportó patología & 3 & $(2,7)$ \\
$(2,7)$ & Gastritis crónica no atrófica & 78 & $(70,3)$ \\
Benignas & Úlcera & 14 & $(12,6)$ \\
$(82,9)$ & Gastritis atrófica & 4 & $(3,6)$ \\
Premalignas & Metaplasia/displasia & 10 & $(9,0)$ \\
y malignas & Cáncer & 2 & $(1,8)$ \\
$(14,4)$ & & & \\
\hline
\end{tabular}




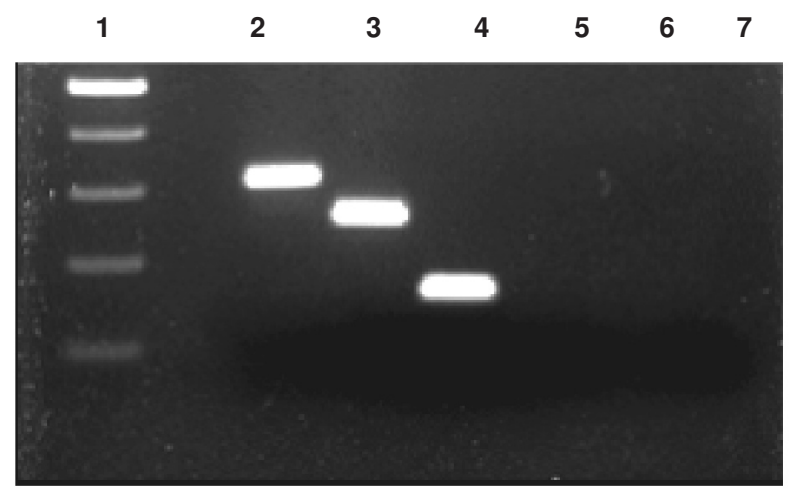

Figura 1. Amplificación de las regiones polimórficas -511, -31 y +3954 del gen de la IL-1 $\beta$ humana: electroforesis en gel de agarosa al 2\%. Carril 1: marcador de peso molecular $100 \mathrm{pb}$ (banda inferior $100 \mathrm{pb}$ ); carril 2: producto de amplificación de la región -511 (305 pb); carril 3: producto de amplificación de la región -31 (248 pb); carril 4: producto de amplificación de la región +3954 (152 pb); carriles 5, 6 y 7: negativos para las reacciones correspondientes.

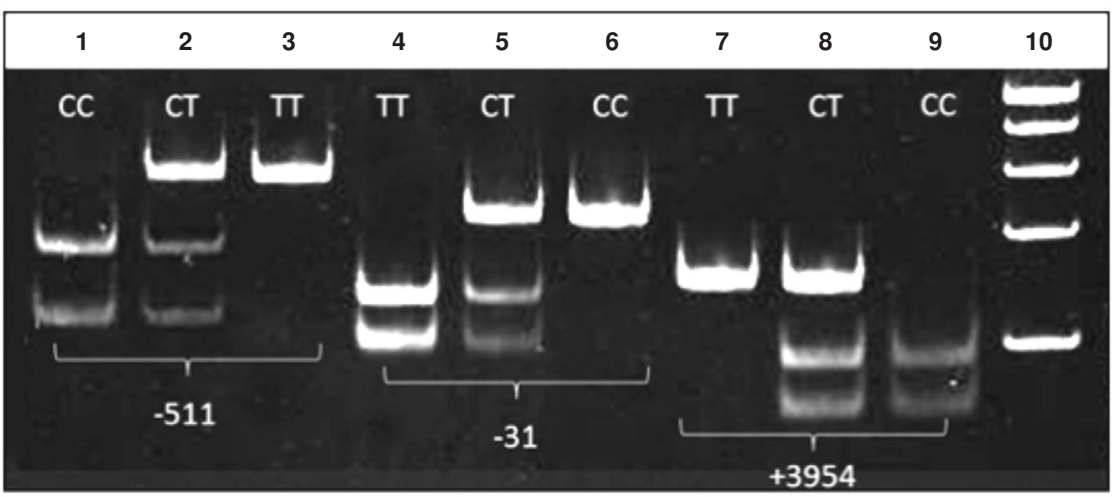

Figura 2. Genotipificación de las regiones polimórficas $-511,-31$ y +3954 del gen de la IL-1 $\beta$ humana: electroforesis en gel de poliacrilamida al $12 \%$. Carriles 1, 2 y 3: muestras de genotipos CC, CT y TT, respectivamente, de la región -511; carriles 4, 5 y 6 : muestras de genotipos CC, CT y TT, respectivamente, de la región -31; carriles 7, 8 y 9: muestras de los genotipos TT, CT y CC, respectivamente, de la región +3954 ; carril 10: marcador de peso molecular 100 pb (banda inferior 100 pb).

Para la posición -511, el alelo C fue observado como dos bandas de 115 pb y 190 pb, respectivamente, mientras que el alelo $T$ fue observado como una banda única de 305 pb. En la posición -31, el alelo $\mathrm{T}$ fue observado como dos bandas de $102 \mathrm{pb}$ y $146 \mathrm{pb}$, mientras que el alelo $\mathrm{C}$ se observó como una única banda de $248 \mathrm{pb}$. Finalmente, para la posición +3954 , el alelo $C$ fue observado como dos bandas de 65 pb y 87 pb, respectivamente, y el alelo T como una banda única de 152 pb (figura 2).

A partir de las frecuencias genotípicas halladas en la población total por medio de un análisis de ji al cuadrado, se determinó que la población de estudio se encuentra en equilibrio de Hardy-Weinberg para las tres posiciones polimórficas evaluadas.

Dentro de la población afectada por las diferentes enfermedades, tanto benignas como premalignas y malignas, se obtuvieron las frecuencias de los genotipos en las regiones polimórficas $-511,-31$ y +3954 del gen de la IL-1 $\beta$ humana, señaladas en el cuadro 2. Debido a la baja frecuencia de las enfermedades malignas y premalignas asociadas a baja secreción ácida, se hizo un análisis dentro del grupo de enfermedades benignas. El cuadro 3 muestra las frecuencias genotípicas de los diferentes polimorfismos, evaluando únicamente los pacientes con enfermedades benignas infectados y los no infectados por $H$. pylori.

Al realizar la comparación entre las frecuencias genotípicas halladas, no se encontraron diferencias significativas en la presentación de los genotipos entre ambos grupos. No obstante, al analizar únicamente los individuos con enfermedades benignas, se encuentra que existe una diferencia significativa entre la frecuencia de presentación del genotipo CC en la posición -31, y se observa una mayor proporción de este genotipo en pacientes infectados con $H$. pylori que en los individuos no infectados con enfermedades benignas.

\section{Discusión}

Debido a las características de la infección por H. pylori, su amplia distribución y las diferentes condiciones patológicas a las que se ha asociado, es de vital importancia su diagnóstico, al igual que el estudio de diversos factores genéticos y ambientales asociados a su huésped. 
Cuadro 2. Frecuencias genotípicas de los polimorfismos de IL-1 $\beta$ según presencia o ausencia de infección por $H$. pylori.

\begin{tabular}{|c|c|c|c|c|c|c|}
\hline \multirow[t]{2}{*}{ Polimorfismo } & \multirow[t]{2}{*}{ Genotipo } & \multicolumn{2}{|c|}{$\begin{array}{l}\text { H. pylori(-) } \\
\mathrm{n}=45\end{array}$} & \multicolumn{2}{|c|}{$\begin{array}{l}\text { H. pylori(+) } \\
\mathrm{n}=66\end{array}$} & \multirow[t]{2}{*}{ Probabilidad } \\
\hline & & $\mathbf{n}$ & (\%) & $\mathbf{n}$ & (\%) & \\
\hline-511 & $\begin{array}{l}\text { TT } \\
\text { CT } \\
\text { CC }\end{array}$ & $\begin{array}{r}10 \\
26 \\
9\end{array}$ & $\begin{array}{l}(22,2) \\
(57,8) \\
(20,0)\end{array}$ & $\begin{array}{l}18 \\
33 \\
15\end{array}$ & $\begin{array}{l}(27,3) \\
(50,0) \\
(22,7)\end{array}$ & $\begin{array}{l}\text { No significativo } \\
\text { No significativo } \\
\text { No significativo }\end{array}$ \\
\hline-31 & $\begin{array}{l}\text { CC } \\
\text { CT } \\
\text { TT }\end{array}$ & $\begin{array}{r}10 \\
29 \\
6\end{array}$ & $\begin{array}{l}(22,2) \\
(64,4) \\
(13,3)\end{array}$ & $\begin{array}{l}19 \\
32 \\
15\end{array}$ & $\begin{array}{l}(28,8) \\
(48,5) \\
(22,7)\end{array}$ & $\begin{array}{l}\text { No significativo } \\
\text { No significativo } \\
\text { No significativo }\end{array}$ \\
\hline+3954 & $\begin{array}{l}\text { TT } \\
\text { CT } \\
\text { CC }\end{array}$ & $\begin{array}{r}0 \\
17 \\
28\end{array}$ & $\begin{array}{r}(0,0) \\
(37,8) \\
(62,2)\end{array}$ & $\begin{array}{c}1 \\
24 \\
41\end{array}$ & $\begin{array}{r}(1,5) \\
(36,4) \\
(62,1)\end{array}$ & $\begin{array}{l}\text { No significativo } \\
\text { No significativo } \\
\text { No significativo }\end{array}$ \\
\hline
\end{tabular}

Comparación de frecuencias: $\mathrm{p}<0,05$

Cuadro 3. Frecuencias genotípicas de los polimorfismos de IL-1 $\beta$ en pacientes con enfermedades gástricas benignas según presencia o ausencia de infección por $H$. pylori.

\begin{tabular}{|c|c|c|c|c|c|c|}
\hline \multirow{3}{*}{ Polimorfismo } & \multirow{3}{*}{ Genotipo } & \multicolumn{5}{|c|}{ Enfermedades benignas } \\
\hline & & \multicolumn{2}{|c|}{$\begin{array}{l}\text { H. pylori(-) } \\
\mathrm{n}=36\end{array}$} & \multicolumn{2}{|c|}{$\begin{array}{l}\text { H. pylori(+) } \\
\mathrm{n}=56\end{array}$} & \multirow[t]{2}{*}{ Probabilidad } \\
\hline & & $\mathbf{n}$ & $(\%)$ & $\mathbf{n}$ & $(\%)$ & \\
\hline-511 & $\begin{array}{l}\text { CC } \\
\text { CT } \\
\text { TT }\end{array}$ & $\begin{array}{r}8 \\
21 \\
7\end{array}$ & $\begin{array}{l}22,22 \\
58,33 \\
19,44\end{array}$ & $\begin{array}{l}16 \\
26 \\
14\end{array}$ & $\begin{array}{l}28,57 \\
46,43 \\
25,00\end{array}$ & $\begin{array}{l}\text { No significativo } \\
\text { No significativo } \\
\text { No significativo }\end{array}$ \\
\hline-31 & $\begin{array}{l}\text { TT } \\
\text { CT } \\
\text { CC }\end{array}$ & $\begin{array}{r}9 \\
24 \\
3\end{array}$ & $\begin{array}{r}25,00 \\
66,67 \\
8,33\end{array}$ & $\begin{array}{l}14 \\
26 \\
16\end{array}$ & $\begin{array}{l}25,00 \\
46,43 \\
28,57\end{array}$ & $\begin{array}{l}\text { No significativo } \\
\text { No significativo } \\
0,0328\end{array}$ \\
\hline+3954 & $\begin{array}{l}\text { TT } \\
\text { CT } \\
\text { CC }\end{array}$ & $\begin{array}{r}0 \\
14 \\
22\end{array}$ & $\begin{array}{r}0,00 \\
38,89 \\
61,11\end{array}$ & $\begin{array}{r}1 \\
23 \\
32\end{array}$ & $\begin{array}{r}1,79 \\
41,07 \\
57,14\end{array}$ & $\begin{array}{l}\text { No significativo } \\
\text { No significativo } \\
\text { No significativo }\end{array}$ \\
\hline
\end{tabular}

Prueba ji cuadrado: $\mathrm{P}<0,05$ significativo

Empleando la técnica de PCR, se detectó $H$. pylori en $59,5 \%$ de los pacientes evaluados con enfermedad gástrica, lo cual es similar a lo anteriormente reportado para ésta y otras regiones colombianas según varios estudios realizados $(9,11,12)$. Bravo et al., en el 2003, estimaron que $61,9 \%$ de biopsias gástricas, aproximadamente, pertenecientes a la población colombiana son positivas, mientras que en lbagué se encontró un porcentaje de infección de $59,5 \%$, el mismo encontrado en el presente trabajo (12).

Es importante resaltar que en los pacientes incluidos en este estudio se encuentran con mayor frecuencia las enfermedades benignas asociadas a niveles de ácido normales o elevados, como son gastritis crónica y úlcera, a diferencia de las enfermedades premalignas y malignas asociadas a niveles bajos de secreción ácida en la mucosa gástrica, como gastritis atrófica, metaplasia, displasia y cáncer gástrico.
Diferentes estudios han señalado que la IL-1 $\beta$ es determinante en el desarrollo de las enfermedades gástricas relacionadas con $H$. pylori, ya que al inducir su expresión como parte de la respuesta proinflamatoria, inhibe fuertemente la secreción ácida en el estómago $(13,14)$, lo cual afecta de modo considerable el desarrollo de la enfermedad. Así, se ha encontrado una correlación entre el aumento en los niveles proteicos de IL-1 $\beta$ y la presencia de algunos de sus polimorfismos genéticos, especialmente de los alelos correspondientes a las posiciones +3954T y $-511 \mathrm{~T}$ del gen (8).

Otros reportes señalan relaciones entre el desarrollo de cáncer gástrico y los bajos niveles de producción de ácido en el estómago con los alelos $-31 \mathrm{~T},-511 \mathrm{~T}(4,6,15,16)$ y +3954 T (5). Además, se ha reportado asociación entre otras enfermedades gástricas, como la gastritis atrófica, con el alelo -31 C (15) y, úlcera duodenal, con el alelo +3954 C (17). En Colombia, al igual que en la población 
italiana, el alelo T en la posición -511 fue asociado con riesgo de desarrollar cáncer gástrico en una población del sur del país $(18,19)$.

Bastante relevante es el papel que juega la IL$1 \beta$ en el desarrollo de la respuesta inflamatoria generada durante la infección por diferentes tipos de microorganismos, lo cual ha sido igualmente reportado en la población colombiana $(20,21)$. En estos estudios se ha visto una relación positiva entre la expresión elevada de la IL-1 $\beta$ y la persistencia y desarrollo de la infección por el microorganismo en estudio, así como del desarrollo de determinado tipo de enfermedad (22).

Sin embargo, aún no se han establecido diferencias entre las frecuencias de alelos en los diferentes polimorfismos entre individuos infectados y los no infectados. Lo anterior se hace evidente, por ejemplo, en varios trabajos sobre la infección por $H$. pylori en la población italiana, los cuales han determinado que portar el alelo $T$ en la posición -511 del gen de la IL-1 $\beta$ incrementa el riesgo de padecer cáncer gástrico; no obstante, no existen diferencias en las frecuencias de los genotipos portadores del alelo entre la población infectada por H. pylori y la no infectada $(19,20)$.

De acuerdo con lo señalado anteriormente, se esperaría que en poblaciones afectadas principalmente por enfermedades benignas, como es el caso del presente estudio, se encontraran en mayor proporción los alelos no asociados a cáncer. Al realizar la comparación dentro de esta población, no se hallaron diferencias significativas entre las frecuencias alélicas de la población infectada por $H$. pylori y la no infectada, lo que sugiere y refuerza la idea de que el genotipo y la respuesta generada por el huésped no determinan en su totalidad el curso de la infección, sino que dependen también, en gran medida, de las características de la cepa infecciosa.

Se encontró que el genotipo CC en la posición -31 se encuentra con mayor frecuencia en la población afectada por $\mathrm{H}$. pylori, lo cual podría indicar que éste genera una predisposición a la infección por H. pylori. Sin embargo, es importante resaltar que estos resultados no apoyan ni contradicen la hipótesis de que esté asociado al desarrollo de cáncer gástrico después de haberse presentado una infección previa por $H$. pylori; además, cabe anotar que el hecho de que esta mayor proporción del alelo $C$ en la posición -31 puede deberse a que, para los análisis, fueran evaluadas únicamente muestras con enfermedad benigna debido a la baja frecuencia de otro tipo de enfermedades, como cáncer, metaplasia y gastritis atrófica (15).

En razón a lo anterior, existe la posibilidad de que, al aumentar el número de muestras de estas enfermedades, se vea un cambio significativo en la frecuencia de presentación de dicho alelo en relación con la infección por H. pylori. También, sería factible que pueda establecerse una relación con el genotipo de los otros polimorfismos evaluados, como se ha comprobado en estudios recientes en los cuales, al comparar las frecuencias genotípicas entre pacientes con cáncer gástrico y gastritis crónica, se determinó que existen diferencias importantes entre las frecuencias de presentación de cada uno de los alelos estudiados (23); o, por el contrario, podría ser que, a pesar de contar con poblaciones comparables, no se encontraran diferencias significativas entre los genotipos, como lo muestran otros estudios (24).

Todas estas observaciones conducen a pensar que existen otros factores importantes en el desarrollo de la enfermedad en el huésped; por lo tanto, en estudios futuros sería interesante, no sólo incluir muestras adicionales de otras enfermedades, sino también, el análisis de otros genes involucrados en la respuesta del huésped.

\section{Agradecimientos}

A los participantes del proyecto "Genotipificación de Helicobacter pylori, caracterización del gen de la interleucina $1 \beta$ humana y su posible asociación con la presentación de determinadas patologías gastroduodenales en pacientes residentes en el Tolima". Al Instituto Nacional de Cancerología, por haber aportado la cepa de referencia de $H$. pylori usada como control en este trabajo.

\section{Conflicto de intereses}

Los autores manifiestan no tener ningún conflicto de interés.

\section{Financiación}

Esta investigación fue financiada por la Facultad de Ciencias de la Universidad de los Andes, Bogotá, D.C., Colombia.

\section{Referencias}

1. Prescott L, Harley J, Klein D. Microbiology. Human diseases caused by bacteria. Sixth edition. New York: McGraw-Hill; 2005. p. 895-96.

2. Dubois A, Borén T. Helicobacter pylori is invasive and it may be a facultative intracellular organism. Cell Microbiol. 2007;9:1108-16. 
3. Scott Algood H, Cover T. Helicobacter pylori persistence: an overview of interactions between $H$. pylori and host immune defenses. Clin Microbiol Rev. 2006;19:597-613.

4. Rad R, Dossumbekoba A, Neu B, Lang R, Bauer S, Saur $D$, et al. Cytokine gene polymorphisms influence mucosal cytokine expression, gastric inflammation, and host specific colonization during Helicobacter pylori infection. Gut. 2004;53:1082-9.

5. Zhang D, Zhens H, Zhou Y, Tang X, Baojun Y, Li J. Association of IL 1 beta gene polymorphism with cachexia from locally advanced gastric cancer. BMC Cancer. 2007;7:45.

6. Zeng Z, Hu J, Hu S, Pang R, Chen M, Sung J. Association of interleukin $1 \beta$ gene polymorphism and gastric cancers in high and low prevalence regions in China. Gut. 2003;52:1684-9.

7. Beales I. Effect of Interleukin $1 \beta$ on proliferation of gastric epitelial cells in culture. BMC Gastroenterol. 2002;2:2-8.

8. Vélez-Castrillón S, Camargo J, Correa P, Anaya J. Bases moleculares de la familia de la interleuquina 1. Revista Colombiana de Reumatología. 2004;11:11-39.

9. Montealegre $\mathbf{C M}$, Jaramillo $\mathbf{C}$, Bohórquez $\mathbf{M}$, Montealegre G, Delgado M. Detección de Helicobacter pylori y caracterización del gen de la interleuquina- $1 \beta$ humana en pacientes de una población colombiana con enfermedades gastroduodenales. Revista Colombiana de Gastroenterología. 2008;23:40-4.

10. Di Giovine FS, Takhsh E, Blakemore Al, Duff GW. Single base polymorphism at -511 in the human interleukin-1b gene (IL1b). Hum Mol Genet. 1992;1:450.

11. Martínez JD, Henao S, Granados C. La gastritis crónica atrófica corporal y la edad. Revista Colombiana de Gastroenterología. 2007;22:17-22.

12. Bravo LE, Cortés A, Carrascal E, Jaramillo R, García LS, Bravo PE, et al. Helicobacter pylori: patología y prevalencia en biopsias gástricas en Colombia. Colombia Médica. 2003;34:124-31.

13. Piñol F, Paniagua M. Citoquinas, gastritis crónica y Helicobacter pylori. Rev Cubana Hematol Inmunol Hemoter. 2000;16:184-9.

14. Fox JG, Wang TC. Inflammation, atrophy, and gastric cancer. J Clin Invest. 2007;117:60-9.
15. El-Omar E, Carrington M, Chow W, McColl K, Yuan C, Rothman $\mathrm{N}$, et al. Interleukin-1 polymorfisms associated with increased risk of gastric cancer. Nature. 2000;404:398402.

16. Chun L, Xia H, Xie W, Ye M, Li J, Cheng H, et al. Association between inteleukin-1 gene polymorphisms and Helicobacter pylori infection in gastric carcinogenesis in a Chinese population. J Gastroenterol Hepatol. 2007;22:234-9.

17. García M, Lanas A, Santolaria S, Crusius A, Serrano M, Peña A. The polymorphic IL-1 $\beta$ and IL-1RN genes in the aetiopahogenisis of peptic ulcer. Clin Exp Immunol. 2001;125:368-75.

18. Palli D, Saieva C, Luzzi D, Masala G, Topa S, Sera F, et al. Interleukin-1 gene polymorphisms and gastric cancer risk in a high-risk Italian population. Am J Gastroenterol. 2005;100:1941-8.

19. Ruzzo A, Graziano F, Pizzagalli F, Santini D, Battistelli V, Panunzi $\mathrm{D}$, et al. Interleukin $1 \beta$ gene (IL-1 $\beta$ ) and interleukin 1 receptor antagonist gene (IL-1RN) polymorphisms in Helicobacter pylori negative gastric cancer of intestinal and diffuse histotype. Ann Oncol. 2005;16:887-92.

20. Flórez O, Zafra G, Morillo C, Martín J, González Cl. Interleukin-1 gene cluster polymorphism in Chagas disease in a Colombian case-control study. Hum Immunol. 2006;67:741-8.

21. Gómez LM, Camargo JF, Castiblanco J, Ruiz-Narváez EA, Cadena J, Anaya JM. Analysis of IL1B, TAP1, TAP2 and IKBL polymorphisms on susceptibility to tuberculosis. Tissue Antigens. 2006;67:290-6.

22. Bartchewsky W, Martini MR, Masiero M, Squassoni AC, Álvarez MC, Sady M, et al. Effect of Helicobacter pylori infection in IL-8, IL-1 $\beta$ and COX-2 expression in patients with chronic gastritis and gastric cancer. Scand J Gastroenterol. 2009;44:153-61.

23. Cañas M, Morán Y, Rivero MB, Bohórquez A, Villegas V, Redón $Y$, et al. Polimorfismo genético de interleuquina-1: asociación con cáncer gástrico en la población de alto riesgo del centroccidente de Venezuela. Rev Med Chil. 2009;137:63-70.

24. Achyut BR, Moorchung N, Mittal B. Genetic association of interleukin-1 haplotypes with gastritis and precancerous lesions in North Indianas. Clin Exp Med. 2008;8:23-9. 